\title{
Distinct ultrafine particle profiles associated with aircraft and roadway traffic
}

Elena Austin ${ }^{\mathrm{a},}$, Jianbang Xianga ${ }^{\mathrm{a}}$, Timothy R. Gould ${ }^{\mathrm{b}}$, Jeffry H. Shirai ${ }^{\mathrm{a}}$, Sukyong Yun ${ }^{\mathrm{b}}$, Michael

G. Yost ${ }^{\mathrm{a}}$, Timothy V. Larson ${ }^{\mathrm{a},+}$, Edmund Seto ${ }^{\mathrm{a},+}$

${ }^{\text {a }}$ Department of Environmental \& Occupational Health Sciences, University of Washington, Seattle, WA 98195, USA

${ }^{\mathrm{b}}$ Department of Civil \& Environmental Engineering, University of Washington, Seattle, WA 98195, USA

*Corresponding Author

${ }^{+}$Co-Senior authors

Total Pages: 9

Table S1. Summary of each mobile monitoring transect

Table S2. Summary of instruments used in the MOV-UP Study

Table S3. Summary of co-location calibration results for PNC and BC monitors.

Table S4. Summary of drive days across the four seasons of the MOV-UP study

Table S5. Summary measures from the mobile monitoring campaign by monitoring location and transect.

Figure S1. Mobile ObserVations of Ultrafine Particles (MOV-UP) Study Setup.

Figure S2. Wind Rose Plots.

Figure S3. Spatial distribution of traffic-related pollutant concentration percentiles

Figure S4. Principal Component factor loadings for each feature of the secondary PCA analysis. 


\section{Tables}

Table S1. Summary of each mobile monitoring transect

\begin{tabular}{|c|c|}
\hline Transects & Range \\
\hline N5 & West Seattle (high-level) Bridge, S. Spokane St., S. Walden St., S. Horton St \\
\hline N4 & SW Holden St., 1st Ave S. Bridge, S. Michigan St., S. Graham St. \\
\hline N3 & SW/ S. 116th St., S. Boeing Access Rd., S. Bangor St., S. Hazel St. \\
\hline N2 & 136th St., S. 135th St., S. 137th St. \\
\hline N1 & SW/ S. 146th St., S. 144th St. \\
\hline S1 & SW/ S. 200th St., S. 199th St. \\
\hline S2 & S. 216th St., 37th P1 S. \\
\hline S3 & S. 240th St. \\
\hline S4 & S. 272nd St., S. 277th St. \\
\hline S5 & SW/ S. 320th St., S. 321st St., S. 319th St. \\
\hline S6 & SW 330th St., S. 336th St., Peasley Canyon Rd S. \\
\hline
\end{tabular}

Table S2. Summary of instruments used in the MOV-UP Study

\begin{tabular}{|c|c|c|c|}
\hline Parameter (units) & Instrument & Manufacturer & Accuracy \\
\hline$\geq 10 \mathrm{~nm}$ Particle Count $\left(\# / \mathrm{cm}^{3}\right)$ & CPC 3007 & TSI & N/A \\
\hline$\geq 20 \mathrm{~nm}$ Particle Count $\left(\# / \mathrm{cm}^{3}\right)$ & P-Trak 8525 & TSI & N/A \\
\hline$\geq 36 \mathrm{~nm}$ Particle Count $\left(\# / \mathrm{cm}^{3}\right)$ & $\begin{array}{l}\text { P-Trak } 8525 \text { with } 36 \\
\text { nm diffusion screen }\end{array}$ & TSI & N/A \\
\hline $\begin{array}{c}\text { 10-420nm Nanoparticle Size } \\
\text { Distributions }\left(\# / \mathrm{cm}^{3}\right)\end{array}$ & NanoScan 3910 & TSI & N/A \\
\hline $\mathrm{BC}\left(\mathrm{ng} / \mathrm{m}^{3}\right)$ & AE51 & AethLabs & $\pm 100 \mathrm{ng} \mathrm{BC} / \mathrm{m}^{3}$ \\
\hline $\mathrm{CO}_{2}(\mathrm{ppm})$ & Li-850 & LI-COR & $<1.5 \%$ \\
\hline Location and Speed & $\begin{array}{l}\text { GPS Receiver DG- } \\
500\end{array}$ & GlobalSat & $\begin{array}{l}\text { Position: }<2.5 \mathrm{~m} \\
\text { Velocity: } 0.1 \mathrm{~m} / \mathrm{s}\end{array}$ \\
\hline
\end{tabular}


Table S3. Summary of co-location calibration results for PNC and BC monitors.

\begin{tabular}{|c|c|c|c|}
\hline Instrument & Intercept* & Slope & $\mathrm{R}^{2}$ \\
\hline P-Trak 1\# & 539 & 0.93 & 0.990 \\
\hline P-Trak 2\# & 197 & 0.97 & 0.992 \\
\hline P-Trak 3\# & -941 & 1.28 & 0.992 \\
\hline P-Trak 4\# & 213 & 0.95 & 0.999 \\
\hline P-Trak 5\# & 127 & 0.90 & 0.974 \\
\hline P-Trak screened 1\# & 386 & 0.87 & 0.966 \\
\hline P-Trak screened 2\# & 372 & 1.04 & 0.955 \\
\hline P-Trak screened 3\# & 194 & 1.05 & 0.948 \\
\hline P-Trak screened 4\# & 673 & 0.89 & 0.987 \\
\hline CPC 1\# & 1208 & 1.20 & 0.993 \\
\hline CPC 2\# & -647 & 0.85 & 0.996 \\
\hline AE51 1\# & 16 & 0.98 & 0.791 \\
\hline AE51 2\# & 143 & 0.89 & 0.932 \\
\hline AE51 3\# & 37 & 1.00 & 0.964 \\
\hline
\end{tabular}

* Intercept units: \#/ $\mathrm{cm}^{3}$ for PNC monitors, and $\mathrm{ng} / \mathrm{m}^{3}$ for BC monitors. 
Table S4. Summary of drive days across the four seasons of the MOV-UP study

\begin{tabular}{|c|c|c|c|c|c|c|c|}
\hline Season & $\begin{array}{c}\text { Sampling } \\
\text { days }\end{array}$ & $\begin{array}{c}\text { Second car } \\
\text { proportion }\end{array}$ & $\begin{array}{c}\text { Start } \\
\text { hour }\end{array}$ & End hour & Temp & RH & $\begin{array}{c}\text { South } \\
\text { Flow } \\
\text { Operation }\end{array}$ \\
\hline Spring & 14 days & $71 \%$ & $11: 00$ & $16: 30$ & $65 \mathrm{~F}$ & $50 \%$ & $52 \%$ \\
\hline Summer & 16 days & $81 \%$ & $11: 00$ & $17: 00$ & $73 \mathrm{~F}$ & $47 \%$ & $75 \%$ \\
\hline Fall & 12 days & $83 \%$ & $11: 00$ & $17: 00$ & $54 \mathrm{~F}$ & $78 \%$ & $91 \%$ \\
\hline Winter & 21 days & $62 \%$ & $11: 30$ & $17: 00$ & $51 \mathrm{~F}$ & $62 \%$ & $59 \%$ \\
\hline
\end{tabular}

Table S5. Summary measures from the mobile monitoring campaign by monitoring location and transect.

\begin{tabular}{|c|c|c|c|c|c|}
\hline Pollutants & Transect & Mean & $\begin{array}{l}\text { Interquartile } \\
\text { Range (IQR) }\end{array}$ & $\begin{array}{c}\text { Standard } \\
\text { Deviation (SD) }\end{array}$ & $\begin{array}{l}\text { Minutes } \\
\text { of Data }\end{array}$ \\
\hline \multirow[t]{13}{*}{ Black Carbon: $\mathrm{ng} / \mathrm{m}^{3}$} & $\mathrm{I}-5$ & 5030 & 3916 & 4319 & 2155 \\
\hline & N1 & 953 & 733 & 1013 & 3935 \\
\hline & $\mathrm{N} 2$ & 909 & 618 & 1133 & 3184 \\
\hline & N3 & 1280 & 972 & 1779 & 3073 \\
\hline & $\mathrm{N} 4$ & 1720 & 1421 & 2504 & 1911 \\
\hline & N5 & 1590 & 1320 & 13867 & 1576 \\
\hline & S1 & 1544 & 1023 & 5149 & 1140 \\
\hline & S2 & 1243 & 985 & 1264 & 1454 \\
\hline & S3 & 1290 & 1068 & 1908 & 571 \\
\hline & S4 & 2832 & 2396 & 13326 & 608 \\
\hline & S5 & 1566 & 1984 & 1545 & 916 \\
\hline & S6 & 3457 & 868 & 1008 & 123 \\
\hline & SR 99 & 2043 & 1992 & 2089 & 431 \\
\hline \multirow{3}{*}{$\mathrm{CO}_{2}: \mathrm{ppm}$} & $\mathrm{I}-5$ & 513 & 55 & 56 & 1640 \\
\hline & N1 & 450 & 40 & 128 & 3306 \\
\hline & $\mathrm{N} 2$ & 434 & 32 & 88 & 2616 \\
\hline
\end{tabular}




\begin{tabular}{|c|c|c|c|c|c|}
\hline & N3 & 456 & 51 & 110 & 2495 \\
\hline & $\mathrm{N} 4$ & 474 & 54 & 135 & 1508 \\
\hline & N5 & 472 & 63 & 115 & 1269 \\
\hline & S1 & 454 & 32 & 161 & 1011 \\
\hline & S2 & 476 & 40 & 190 & 1217 \\
\hline & S3 & 462 & 26 & 170 & 528 \\
\hline & S4 & 485 & 38 & 162 & 559 \\
\hline & S5 & 468 & 36 & 77 & 783 \\
\hline & S6 & 443 & 24 & 19 & 123 \\
\hline & SR 99 & 480 & 66 & 155 & 349 \\
\hline \multirow{13}{*}{$\begin{array}{c}\text { Total UFP (10-1000 } \\
\mathrm{nm}): \# / \mathrm{cm}^{3}\end{array}$} & I-5 & 59896 & 41833 & 37704 & 2121 \\
\hline & N1 & 20160 & 18022 & 16555 & 3853 \\
\hline & N2 & 18318 & 15581 & 15260 & 3161 \\
\hline & N3 & 20124 & 16747 & 18975 & 3184 \\
\hline & N4 & 23186 & 16487 & 20715 & 1917 \\
\hline & N5 & 19868 & 14132 & 19123 & 1606 \\
\hline & $\mathrm{S} 1$ & 16340 & 12139 & 21452 & 1139 \\
\hline & S2 & 19150 & 15202 & 16831 & 1430 \\
\hline & S3 & 13433 & 8056 & 14088 & 567 \\
\hline & S4 & 18723 & 13787 & 19496 & 606 \\
\hline & S5 & 14500 & 10819 & 11427 & 903 \\
\hline & S6 & 9713 & 5589 & 4402 & 123 \\
\hline & SR 99 & 26117 & 19768 & 21077 & 407 \\
\hline
\end{tabular}




\section{Figures}
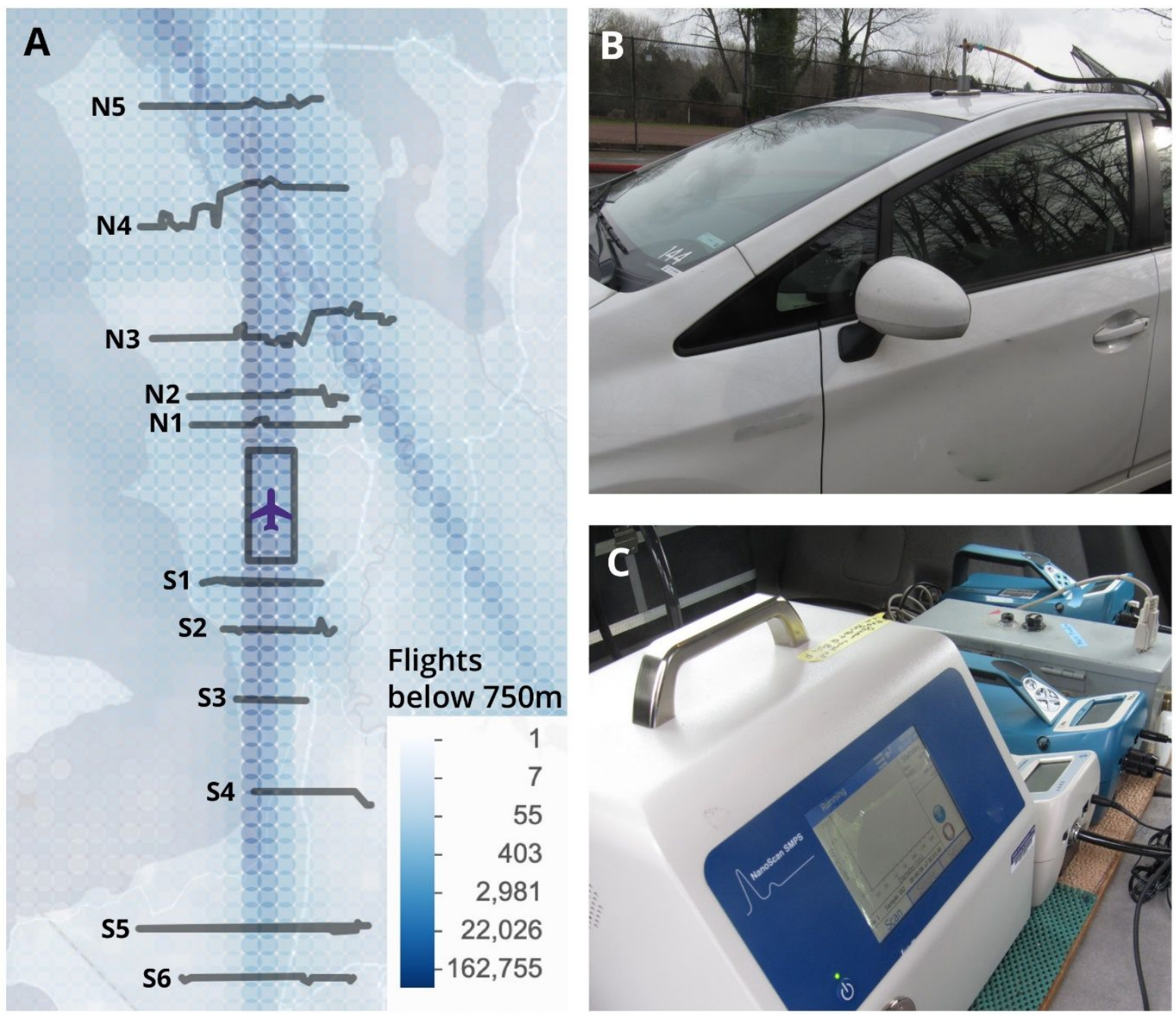

Figure S3. Mobile ObserVations of Ultrafine Particles (MOV-UP) Study Setup. A. Displayed on the map are the location of the 5 transects North of the airport, labeled N1-N5, and the 6 transects South of the airport, labeled S1-S6. In blue, the density of flights at an altitude of $750 \mathrm{~m}$ or less is overlaid on the street map. B. Mobile platform with rooftop air inlet. C. Sampling manifold and monitoring instruments. 
Frequency of counts by wind direction (\%)
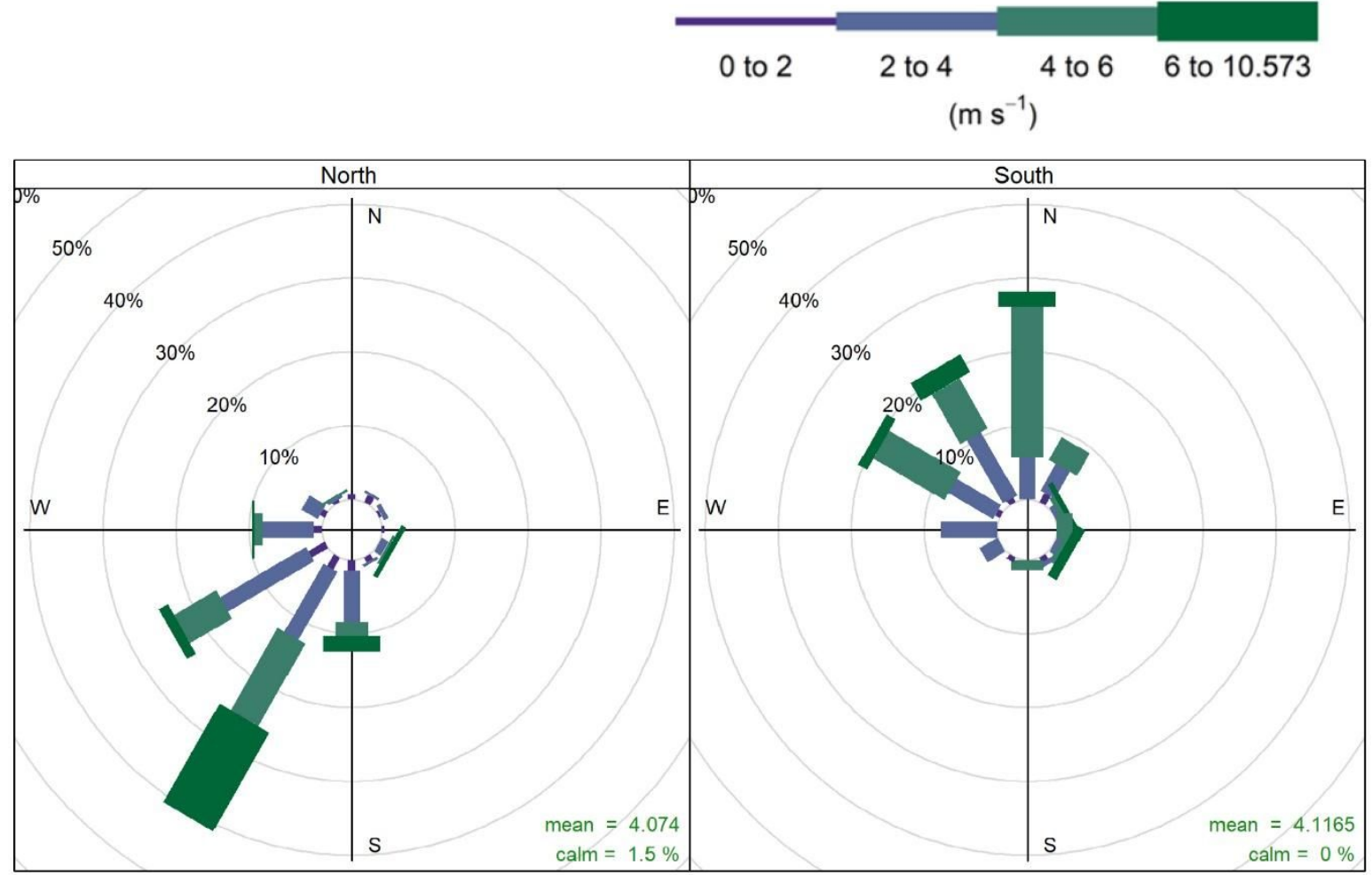

Figure S4. Wind Rose Plots. Represents the wind speed and direction over the course of all mobile monitoring sampling campaigns, separated by the landing direction of the aircraft. The north and south labels on the plot refer to the direction from which aircraft were landing with respect to the airport. 

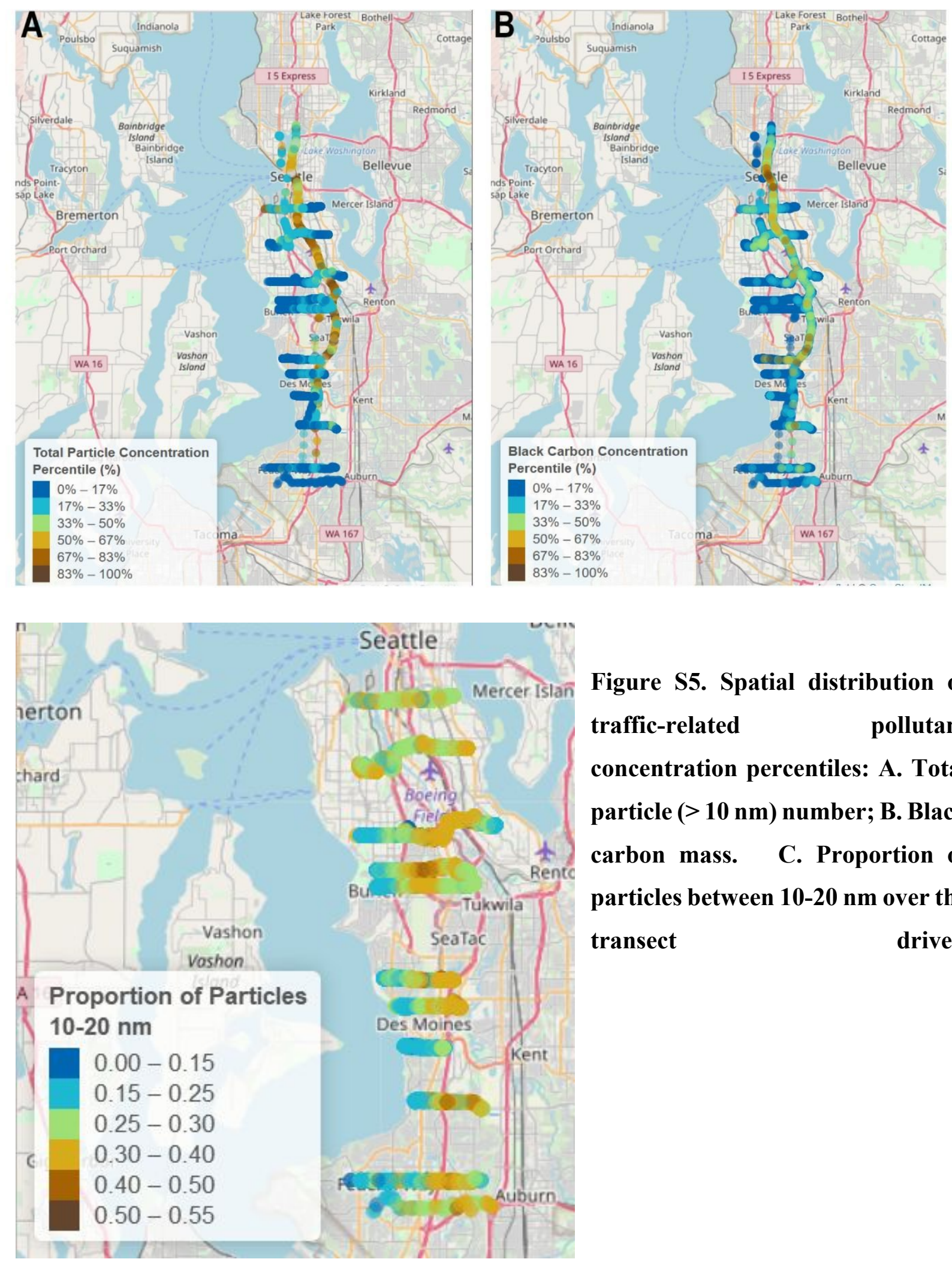

Figure S5. Spatial distribution of traffic-related pollutant concentration percentiles: A. Total particle (> $10 \mathrm{~nm}$ ) number; B. Black carbon mass. C. Proportion of particles between 10-20 $\mathrm{nm}$ over the transect drives. 

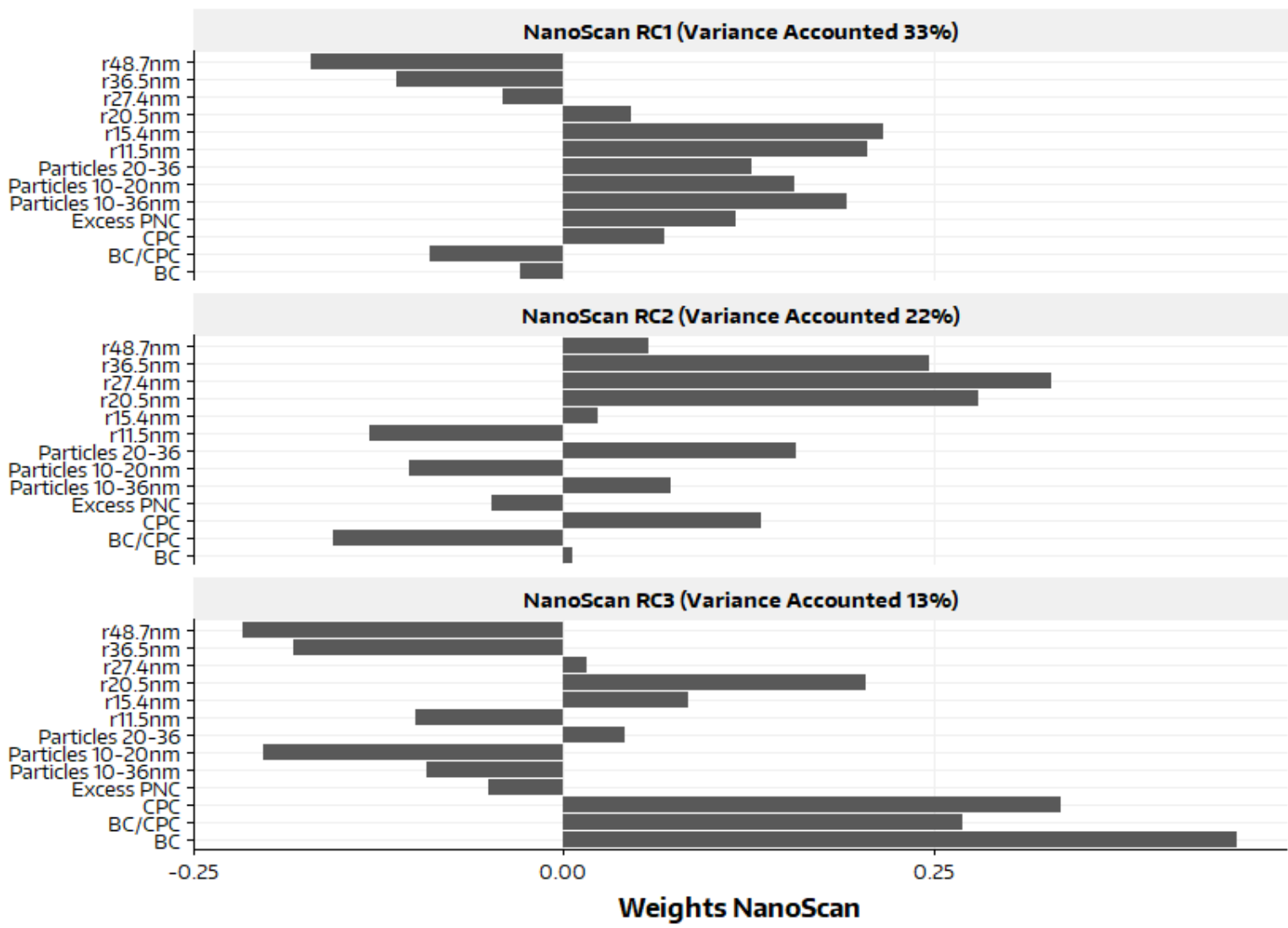

Figure S6. Principal Component factor loadings for each feature of the secondary PCA analysis. 


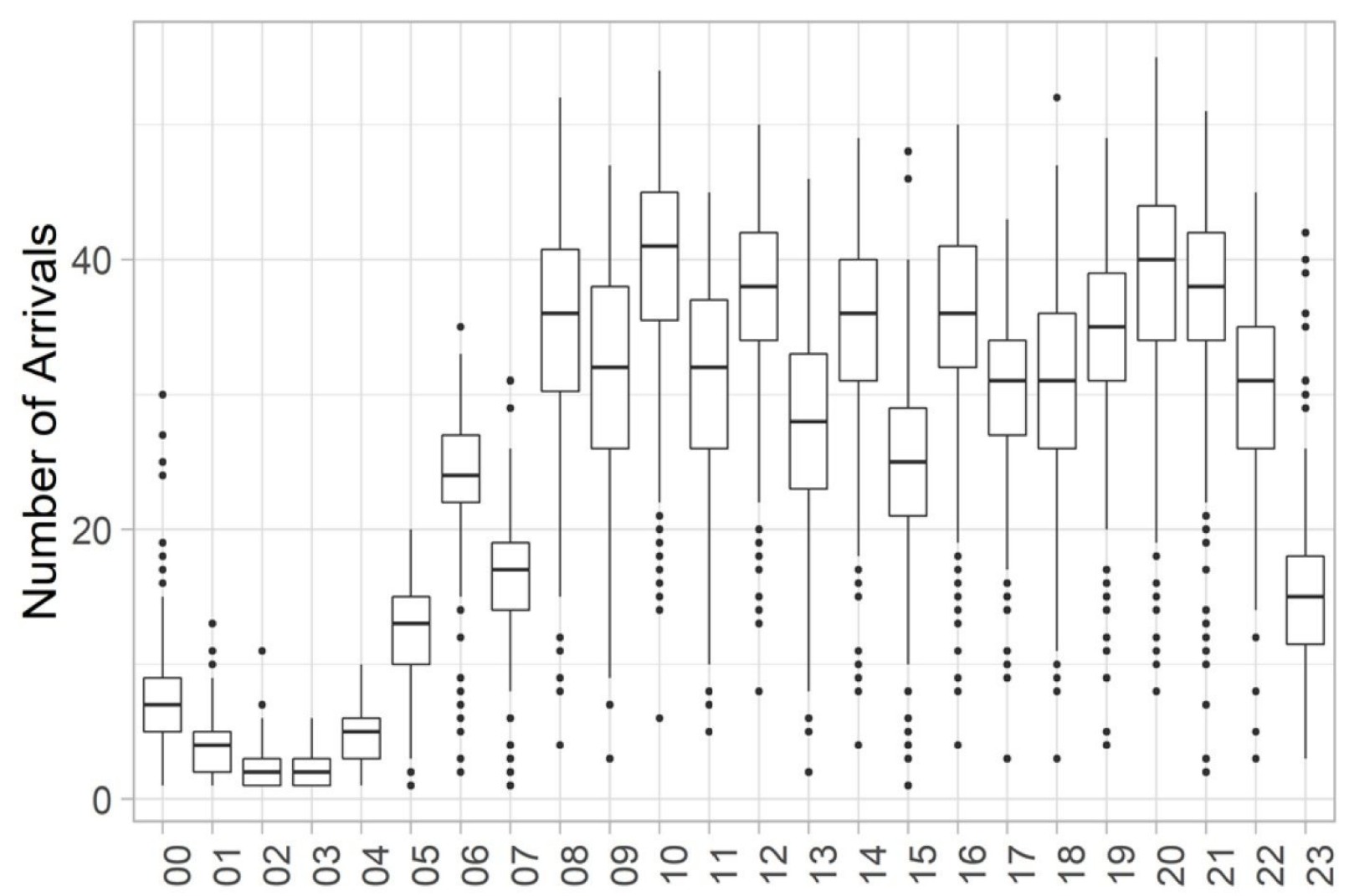

Figure S5. Diurnal patterns in SEA-TAC arrivals over 2018. 